\title{
Editarial
}

\section{Ending elitism in peer-review publication}

\author{
Lonnie W. Aarssen and Christopher J. Lortie
}

L.W. Aarssen (aarssenl@queensu.ca), Dept. of Biology, Queen’s University, Kingston, ON, Canada, K7L 3N6

C.J. Lortie (lortie@yorku.ca), Dept. of Biology, York University, Toronto, ON, Canada, M6S 2E2

The peer-review publication process can be used to assign credibility, improve dissemination, and as a proxy to compare the relative merits of studies. Researchers, however, remain frustrated by limitations that continue to escape solutions (e.g. Alberts et al. 2008). Partly because of these challenges, IDEAS IN Ecalogy AND Evalutian was launched as an experimental model for exploring changes to the conventional process. The journal has now completed its first full year, with three high quality papers published in 2009. Submission rate was low (one additional submission was rejected), but we anticipate that the exposure proffered from volume 2, our efforts to promote the journal over the past year, plus recent changes to author fees, will generate a growing submission rate through 2010. Several novel features were incorporated initially (Aarssen 2008) in our enthusiasm to address several issues all at onceprobably too many, as it turns out, and so our experience in this past year has called for some revised perspectives, detailed below in the context of re-visiting the mission of the journal.

IEE was developed in response to three conspicuous problems associated with peer-review publication:

(i) There is an intrinsic bias against the publication of new ideas. In most traditional journals, there is limited interest in publishing 'ideas' or 'commentary'-style papers. And because of high rejection rates in many journals, it can often take more than a year to get a new idea published, by which time it may already be oldscooped by someone else. An alternative is to promote ideas through blogging, but most scientists don't blog because they receive no credit or recognition for blogging (Waldrop 2008). IEE is the only peer-review journal for ecologists and evolutionists that is dedicated exclusively to 'forum'-style papers. It has a completely transparent and objective protocol for manuscript acceptance/rejection [Fig. 1 in Aarssen (2008)] that guards against referee bias, and serves to break down traditional barriers to the release of creativity. IEE embraces the perspective offered recently by Gardner et al. (2007: 510): “Although the process of using ideas to generate an hypothesis is less structured than the rulebased methodological testing of that hypothesis it is nonetheless integral to the scientific process. [...] The generation of an hypothesis is analogous to the search for 'truth' in history. Historians examine and compare narratives to synthesize an accepted version of truth, and after scrutiny such stories might become accepted as fact.”

(ii) It is increasingly difficult to attract referees, and to obtain high quality, objective reviews. As the lives of potential referees keep getting busier, there is less and less motivation for gratuitous reviewing. Finding people who have the time to review, therefore, is a growing challenge for editors. And when found, the reluctant, over-worked (and unhappy) referee often presents arbitrary, debatable, poorly argued, biased, and/or draconian recommendations for rejection or major revisions. With little incentive to review, quality suffers accordingly, and referees can get away with mediocre and biased reviews because they can hide behind anonymity, with no accountability to anyone. This must end, and rewards and transparency are by far the most effective means for change.

The philosophy of IEE is that referees are analogous to expert consultants/witnesses in a court of law, who are often paid for their professional assessment and are always accountable for their views, and hence never anonymous. Accordingly, we launched IEE with the 
goal of regarding referees as paid professionals that are identified within published papers; i.e. with no anonymity. The reasoning is that paying referees who are acknowledged within the publication should improve both the motivation to review, and the incentive and accountability for providing high-quality, objective reviewing. The benefits of reviewer accountability for the author (and the progress of science) should outweigh the potential cost to the reviewer from possible author retaliation for a negative review. Moreover, with consistent and universal referee acknowledgement, accountability would be in theory enforced for everyone, thus inhibiting retaliation by everyone (because there would be no anonymity for anyone to hide behind). Although this model requires a submission fee from authors, the money is returned to the community of colleagues (as remuneration for reviewing), rather than paid to support profits for big publishers. Why should businesses incur financial gain from the efforts of researchers to secure their own research funding, conduct the research, then review each others' work and publish it? This is completely unnecessary in the age of electronic communication and publication.

In our consultation with colleagues over the past year, most favoured the idea of getting paid to review, but unfortunately, few liked the idea of having to pay the submission fee necessary to have this service applied to their own papers. This model presents a deterrent particularly for people with little or no research funding (e.g. graduate students, post-docs, many researchers in less developed countries) who are especially likely to avoid a system that requires 'upfront' charges that could end up not yielding benefits. Accordingly, there are now no submission fees for IEE, and consequently, referees will not be paid. Each paper is presently charged a fixed publication fee of CAN $\$ 300$ only after it is accepted for publication. This is less expensive than any other open-access journal. IEE uses this fee only to recover the basic operating costs of the journal-e.g. handling and correspondence time, copy editing, proof production and publication, website maintenance, PayPal service, and DOI registration. The publication fee will be adjusted only if necessary for the journal to continue operation on a cost-recovery basis.

Submission charges for paying reviewers may have merit someday, but apparently not yet, or at least not without instituting a funding mechanism that could support waiving of submission fees based on financial need. For now, apparently, the growing erosion of reviewer incentive remains an ongoing problem for peer-review publication.

(iii) There is now a rampant and crippling culture of elitism centered on journal impact factor. Science is a mission for discovery-nothing else. Yet many editors and publishers in science journalism have become more concerned about a mission for elitism and profit, associated with boosting journal impact factor. To accomplish this, editors of some journals now routinely reject good-quality manuscripts that are rated highly by referees, and in some cases without even seeking referee assessment. They 'justify' this by claiming to have limited page numbers for each volume of the journal, and so allegedly there is space to publish only the 'best of the best'. This is a sham, and presents an opportunity to camouflage publication bias. Virtually everyone now reads from digital/electronic production, including students in university libraries, where paper-based journal subscriptions are being cancelled at faster rates with each passing year. Page-space limitation therefore no longer exists literally, yet many publishers and editors continue to embrace it pretentiously, as an absurd gate-keeping mechanism for protecting and promoting elitism. Because the power here rests with publishers and editors, many authors-especially in early career stages_-are left with no option but to yield to the addiction of chasing impact factor in efforts to achieve career advancement. This is a waste of time for everyone involved in the system, not just the authors. These authors believe that their papers will be highly regarded (e.g. in considerations for tenure review and grant applications) just because they appear in high impact factor journals-even though many papers in these journals receive low citation rates (Koricheva and Leimu 2005). This obsession with impact factor has produced disillusionment and inefficiencies (Campbell 2008, Raff et al. 2008, Simons 2008), and even academic misconduct (Martinson et al. 2005, Titus et al. 2008, Young et al. 2008) in the practice of science. The purpose of publication is to disseminate publicly funded research, not to seek an impact factor branding 'label' in order to make a statement that one's work must therefore be better than others.

In contrast, manuscript rejection in IEE is never based on page-space limitation; publication is on-line only. Rejection is decided only on the basis of transparent and objective criteria, and is never based on elitist goals to publish only the 'best of the best'. The best papers in IEE will earn their own merit and recognition honestly through their own citation frequencies.

In conclusion, IEE extols three guiding principles for breaking free of the mission for elitism in peer-review publication:

(a) The quality of one's paper should be judged by earned merit (e.g. the number of citations, website accesses, or downloads)—not by the impact factor of the journal in which it gets published. Thankfully, university administrators and research granting agencies are starting to show signs of doing this. Few academics 
are comfortable with being labeled as elitist, and so there may be a growing sentiment to correct this problem. Science progresses through discovery, not through the elevation of a journal's impact factor. Elitism has no place in science, and reducing the power of impact factor elitism will help to curb academic misconduct and publication bias.

(b) Authors now have free and immediate access to citation metrics regardless of where their papers are published. Google Scholar now tracks citations and will list them at no charge. Other competitive citation services (e.g. Scopus) have also been developed recently. New open-access journals like IEE, therefore, no longer must wait for the long 'qualifying period' (often years) imposed by ISI Web of Science before it will index a new journal. Now an author who publishes in a new open-access journal like IEE can immediately and freely obtain a direct metric of the paper's merit from Google Scholar. Access and download statistics are also becoming more readily available from journal websites as meaningful measures of merit.

(c) Because of internet technology (freely available to most academics), an author who publishes in a new open-access journal like IEE can easily ensure that the paper is readily and quickly visible to colleagues around the world. Once a paper is published, authors can now quickly promote it without any cost, and with very little effort - for both authors and readers. This is easily accomplished by sending an email notice to all (or the majority of) published authors in one's field, including a cut/paste of the abstract, together with the URL for free download of the article from the journal's website. One can generate a handy email list for this after an hour or so of searching on the internet. Then, with every new paper that one publishes in a new openaccess journal like IEE, just a few mouse clicks will send it straight to the desktop of most published authors that will be interested in it, thereby increasing the likelihood of citation. Newly published papers can also be promoted widely now through blogs and list-serves such as ECOLOG.

Publishing in a high impact factor journal, therefore, is no longer necessary in order to promote the visibility of one's paper. Of course it is true that papers published in these journals traditionally have always attracted some automatic attention, even before they are read. But prior to the availability of digital/electronic online publication, this effect of high impact factor was an entirely reasonable consequence of legitimate competetion for limited space on real pages within paper volumes. Today however, with paper volumes now going extinct, the attention and status associated with publication in a high impact factor journal is largely a product of gate-keeping exclusion strategies based on a fictional competition for limited page-space that exists only as pretence in the minds of elites. The editors of IEE invite ecologists and evolutionists to participate in this experiment-let's end elitism in peer-review publication, minimize publication bias, and be more creative, not only in our ideas in ecology and evolution but also in how we review them.

\section{Acknowledgements}

Jennifer Waugh provided her usual sage guidance and advice in editing the manuscript.

\section{References}

Aarssen, L.W. 2008. Ideas in Ecology and Evolution A new open-access model dedicated to the rapid release of creativity in peer-review publication. Ideas in Ecology and Evolution 1: 1-9. CrossRef

Alberts, B, Hanson, B. and K.L. Kelner. 2008. Reviewing peer-review. Science 321: 15. CrossRef

Campbell, P. 2008. Escape from the impact factor. Ethics in science and environmental politics 8: 5-7. CrossRef

Gardner, J., Marsack, P., Trueman, J., Calcott, B. and R. Heinsohn. 2007. Story-telling: an essential part of science. Trends in Ecology and Evolution 22: 510. CrossRef

Koricheva, J. and R. Leimu. 2005. What determines the citation frequency of ecological papers? Trends in Ecology and Evolution 20: 28-32. CrossRef

Martinson, B.C., Anderson, M.S. and R. de Vries. 2005. Scientists behaving badly. Nature 435: 737-738. CrossRef

Raff, M., A. Johnson, and P. Walter. 2008. Painful publishing. Science 321: 36. CrossRef

Simons, K. 2008. The misused impact factor. Science 322: 165. CrossRef

Titus, S.L., Wells, J.A. and L.J. Rhoades. 2008. Repairing research integrity. Nature 453: 980-982. CrossRef

Waldrop, M.M. 2008. Science 2.0 - Is open access science the future? Scientific American. http://www.sciam.com/article.cfm?id=science-2point-0

Young N.S., Ioannidis, J.P.A., and O. Al-Ubaydli. 2008. Why current publication practices may distort science. PLoS Med 5: e201 $\underline{\text { CrossRef }}$ 